\title{
Knowing and learning in everyday spaces (KALiEds): Mapping the information landscape of refugee youth learning in everyday spaces
}

Journal of Information Science

$1-13$

(C) The Author(s) 2015

Reprints and permissions: sagepub.co.uk/journalsPermissions.nav DOI: $10.1177 / 0165551510000000$ jis.sagepub.com

\author{
Annemaree Lloyd \\ School of Information Studies, Charles Sturt University, Wagga Wagga, New South Wales, Australia \\ Swedish School of Library and Information Studies, University of Borås, Sweden.
}

\section{Jane Wilkinson}

Educational Leadership, Faculty of Education, Monash University, Clayton, Victoria, Australia

\begin{abstract}
Refugee youth are faced with complex information needs that require them to identify and map the everyday spaces that can contribute to their learning outside the formal schooling system. The use of everyday spaces by refugee youth aged $16-25$ was investigated using photovoice and interview data collection methods. The findings of the study suggest that the information needs of this cohort arise from the desire to connect with a new community, to learn new social rules, while at the same time supporting the information needs of other family members and dealing with the social challenges that arise from cultural expectations. These challenges require them to connect with a wide range of everyday spaces to support their learning needs.
\end{abstract}

\section{Keywords}

Information literacy, informal learning, everyday spaces, refugee youth.

\section{Introduction}

Research highlights that refugee youth in the 16-25 year age bracket who have been forced to resettle frequently have experienced disrupted educational backgrounds that may create disadvantages and have implications for their educational or employment prospects in their new country[1,2]. To accommodate and meet the challenges that exist outside the formal compulsory and post-compulsory education systems, refugee youth must identify and use a wide range of everyday spaces to connect with information to satisfy their own learning needs, and to support the needs of their families. Recent studies [1,2] of refugee youth suggest that in regional centres, informal everyday learning spaces such as libraries, community interest groups, and/or faith-based groups provide a valuable opportunity for these young people to build the social and cultural capital, information and other literacies required to access the often 'invisible', more hidden systems of access.

The findings of the study which are described in this article, suggest that everyday spaces act as rich sites of informal learning, and young refugee people draw upon these sites and knowledges to enhance their ability to gain entry to the established community and to link into a wide range of prospects that enable them to position or situate themselves in relation to opportunities such as finding a job, learning new skills, or simply fitting into the community. Encountering an everyday space, places a person temporally and spatially, positioning them in relation to the practice arrangements or architectures that shape the sayings, doings, and relatings of a site [3,4]. These architectures enable and/or constrain the normative sources of information and also prefigure (but do not predetermine) the encountering and recognition of nonnormative information sources [4]. Normative sources refer to the information resources that relate to social rules, regulations and prescribed ways of knowing that enable communities to operate and people within the communities to 
learn how to go on. Non-normative sources refer to the insider or internal views and represent nuanced or embodied aspects -they can consequently be viewed as local /vernacular and imbued with emotional resonance [5]. A significant feature of the current everyday spaces study is the breadth and complexity of explicit, implicit and contingent sources of information that are present. Consequently, refugee youth must develop their information literacy practice, described by Lloyd [3, p. 26] as ' a way of knowing the many environments that constitute an individual being in the world' to support their learning. Gaining access to information affordances of these spaces, enabled refugee youth to transition into the community and become emplaced.

A socio-theoretical approach influenced the research design and analysis. This approach drew from and combined interdisciplinary perspectives in information studies and education $[6,7,1]$. Theories of social interaction [8], of social capital [9], of practice [10], and practice architectures [4, 3] were also drawn upon to guide the analysis. The question of how everyday spaces may be supporting the information needs, literacies and learning practices of this cohort of regional refugee youth while simultaneously building social inclusion, has not been previously considered. The present study will contribute knowledge that addresses this gap by exploring how participants engaged with everyday spaces. This paper will introduce the research context, methodology and findings of the phase one analysis of young people's information use in support of their learning in everyday spaces.

\section{I. Background: Everyday spaces and information landscapes}

A comprehensive analysis of the literature that synthesises the concept of everyday spaces is beyond the scope of this paper. It is suffice to say that the concept of everyday spaces is rarely described in the Library and Information Science literature, even though it is referenced often $[11,12,13]$. In the Library and Information Science field, the concept of everyday spaces appears comparable with the concept of everyday life used in the social sciences. In this field, everyday life is well developed, particularly through social phenomenology $[13,14]$ and critical theory [15].

Investigations which have produced a construct of space have emerged from philosophy through the work of Lefebvre [16] and Schatzki [17]. Lefebvre drew from Marxian tradition to describe everyday spaces as "whatever remains after one has eliminated all specialised activities"; that is, evoking a sense of ordinary spaces and territories where routine, informal daily life occurs. In describing social space, Lefebvre conceptualised that social space was constituted through a trifold representation. This comprised perceived space where human activity is performed, conceived space that acts as a non-symbolic, non-representation of space, and lived space representing symbolic, emotional performances [16]. Schatzki [17], amalgamates this trifold representation to draw perceived and lived space together, arguing that the physical body and nature are represented in perceived space. He contends that "This space is perceived and acted in by both actors and those who observe their activity." Further Schatzki observes that "People also live through perceived spaces.... these spaces are meaningful physical spaces" [17, p.15].

Other ways of viewing everyday spaces have also emerged. In a study of development-displaced migrants in India, Chattopadhyay [18] divides 'everyday spaces' into four thematic groups: lost, rehabilitated, contested and personal spaces. Lost spaces refer to migrants' lives in their submerged villages. Rehabilitated spaces are generated in the resettled villages and are currently lived. Contested spaces often arise from counter-discourses, confrontation and feuds at the community level, pertaining to the control and use of resources in rehabilitated villages. Personal space describes emotions, activities, household roles and relationships [18, p. 86]. These conceptualisations present 'spaces' as more than physical locations, but also as arenas of thought, emotion, activities, roles and relationships.

The concept of everyday spaces presented here is informed by this literature and draws from an information perspective, which focuses on information as the core element central to knowledge construction and the need to obtain information as the catalyst for learning. Implied in this statement and elaborated elsewhere [3] is Bateson's [19, p. 1972] view of information 'as any difference that makes a difference", which suggests that social relationships, connectedness and situatedness are necessary conditions to realise data into information and information into knowledge.

Everyday spaces can therefore be defined by their information characteristics, and the information resources they afford. They have physical, affective and cognitive resonance for those who inhabit or recognise it and reference the shared common understandings of people involved in similar endeavours or performances (e.g., playing a game, learning a skill). Everyday spaces may therefore be physical, e.g., a location, they may be virtual, or emotional, but they exist temporally and spatially. Each space entwines with others to build the information landscape which is shared by people who are engaged in the project of mutual understanding [20,3].

A landscape reflects and emphasises the ongoing interactions of people with the socio-cultural, historical, political and material-economic conditions of their setting. There is an emphasis on the construction and shaping of knowledge 
which influences shared ways of understanding $[8,21,5]$ and meaning making. Information landscapes represent knowledge spaces that have resonance with people who are involved in collective practices, endeavours or situations $[20,3]$. A landscape therefore represents the total relations that exist between people who engage in similar performances [22, 21] and through those performances develop similar ways of knowing and making meaning. Information landscapes are shaped via information modalities that have resonance with the site through performance, endeavour or situation [20]. The landscape is therefore formed and accessed through the semantic space (through language), materially (through analogue and digital mediums; through the use of material objects) and corporeally through performance and observation of performance (embodied practices). Information landscapes can therefore be viewed as comprising complex multiple spaces each entwined in a complex information ecology [23] which frames particular discourses and narratives and thus entwine people within time and space. In the present study the construct of the information landscape informed the methodology which is described below.

\section{Aim, Methodology, and Research Design}

The Knowing and Learning in Everyday Spaces (KaLiEDS) project aimed to document and map how refugee youth are engaging in everyday learning spaces, and to describe and analyse the information needs, literacies and learning practices used by refugee youth (aged 16-25 years) located in a regional city in Australia. The primary question posed by the study was:

How are everyday spaces employed by refugee youth to support the development of information literacies and learning practices?

Sub questions that unpacked this primary question, focused on the types of information, literacies and learning employed by participants, how these literacies were meeting information needs, how access to information was managed, and the types of affordances furnished through everyday spaces. A significant aspect of this study was to understand what the information landscape looked like for refugee youth who participated in the study. Specifically, the role and affordances of everyday spaces as information sources were explored and mapped to understand how spaces were experienced and contributed to informal learning. This aspect will be reported on in this paper.

\subsection{Research Protocol}

Research that involves young people from CALD (Culturally and Linguistic Diverse) backgrounds and the use of visual techniques such as photovoice presents a number of complex issues. These required the development of a number of protocols to ensure that participants had a clear comprehension of the project; were aware of their own personal safety; and also developed an understanding of how to take photos while remaining respectful to members of the public.

\subsection{Ethics of working with CALD Groups.}

The research was conducted in English, with participants who had English as another language. To assess comprehension of the study, potential participants had the project explained to them in detail and then were asked to describe the tasks and activities they would be engaged in during the study. Participants were asked to describe in plain English the role of the study. This allowed the researchers to determine whether language and comprehension was sufficient enough for participation. This process also allowed the Chief Investigators to determine whether potential participants were of sufficient maturity to participate.

\subsection{Sample, training workshops and data collection}

The sample for this study was 15 refugee young people between the ages of 16 and 25 years of age. The participants were recruited via a number of support agencies. The majority of participants were from a range of African nations, including Sudan, with three participants from Afghanistan. Of the group, twelve participants were male and three female. Numerous attempts were made to increase the number of females in the sample, but these were not successful. This could be viewed as a limitation of the study. 


\section{I.4. Method}

For many people the concept of information is often difficult and intangible, which makes it a difficult thing to describe [21]. This challenge can be further compounded when English is not their first language. To address this issue, photo voice technique was employed and coupled with focus groups, allowing participants to visually capture information or representations which reflected their understanding of what information was meaningful to them and contributed to their learning.

\section{I.4.I. Photovoice technique}

Photovoice technique is becoming increasingly important to the social science methodology toolbox and has significant benefits for research in the Library and Information Sciences field. The technique allows participants to represent aspects of their everyday life that are often difficult to articulate (e.g. what types of information are important to them, where do they find it). In the Library and Information Science field, the technique has been employed by Julien, Given and Opryshko [24] who utilised the technique in a study of high school students' information literacy skills as they transfer from high school to university. Lloyd has also employed and discussed the merits of this approach [5] in relation to following the red thread of information use. In education, Wilkinson has also employed the technique [1].

The photovoice method aims to empower participants to take control of the research process and in doing so gives them power to develop narratives about what counts and to represent that through visual means using photographs as a visual information tool. The methodology was developed by [25] and in the years since has been implemented in a wide range of studies and with a wide range of populations. The methodology has been closely associated with health promotions. Underpinning the methodology are theoretical perspectives associated with Freire's critical consciousness, and with feminist theory $[25,26]$. There are three main goals associated with the photo voice technique. These are to:

- allow participants to record and reflect on issues and concerns;

- promote critical dialogue and knowledge about a range of issues; and,

- reach policy makers.

Benefits of this technique are:

- validation of participants' 'voice';

- ability for participants to see a range of views associated with same issue;

- increased awareness of issues; and,

- effect social change $[25,26]$.

A significant feature of the photovoice method is that the photos that are elicited and selected by participants act as a tool for deep engagement in the research process. Importantly, the use of this technique provides participants with control over how data is generated, what data is generated, thus allowing them more control of the research. In Library and Information Sciences research, the benefits of this particular method rest with participants being able to photograph types of information, information related situations and sources that are important to them, rather than having to describe them.

\subsubsection{Limitations}

While the technique provides a powerful tool to be included in Library and Information Sciences research toolbox, there are also limitations to the technique. These were related to the quality of cameras provided, especially given that most participants owned their own mobile phone which often had much better camera quality. A second limitation related to ensuring that participants could articulate clearly why a certain photo was included in the research sample, and that necessary permissions had been signed.

\section{I.4.3. Photography workshop and focus group session}

Three workshops were held as part of the research project. In the first workshop participants were given training in the use of cameras and the protocols they were required to adhere to when taking photos. Topics covered in the workshop included not taking risks when photographing and ensuring that permissions were obtained prior to taking photographs. At the conclusion of the workshop, participants undertook a small photography exercise, which comprised taking a few photographs and then returning to the workshop to discuss and reflect on the process. 


\subsubsection{Photography phase}

At the conclusion of workshop one, participants were then given the task of photographing the types of information and information sources that were important to them and the places where that information was located. It is interesting to note that all participants were familiar with digital photography as cameras are a standard inclusion in most mobile phones. During the training session it was therefore important that researchers emphasised the focused nature of data collection to ensure that all participants remained aware of the type of photos that were required. During the photography phase the researchers remained in regular contact with participants via a social media site to remind them of the purpose of the study, to ensure a connection between the researchers and the participants and that participants remained committed to completing the project.

\subsubsection{Second Workshop}

A series of second workshops were held over several weeks to ensure that all participants were able to attend. Participants returned to the second workshops with the five photos each had selected for the study. Each participant was asked to describe their photographs, and then asked a series of questions about what the photograph represented, what sources of information were being identified, why the spaces were important to their learning and what learning was taking place. Other members of the focus group were asked to comment on the photos taken by each participant and discussion was then held following the themes of information sources, everyday spaces and learning. This technique provided both emic (participant insider) and etic (participant outsider) perspectives to be incorporated into the data sample [5].

\subsubsection{Final workshop}

A final workshop was held prior to project delivery to the community. During this workshop, the situational map of information sites and sources and analysis of interview data was presented to the participants. This ensured the veracity of the analysis and approach represented participants' understanding of the project outcomes and also ensured that key messages to stakeholders groups were formalized and approved by the participants.

\section{I.4.7. Focus groups}

Focus groups were employed to support the photovoice technique. The focus groups provide a more informal and collaborative setting, affording participants the opportunity to share photos and reflections with other members of the research group and allowed them to explore a range of participants' perspectives [27]. Focus groups were held on a number of days to accommodate participants' schedules. The size of each group varied between 3 and 5 participants.

Participants controlled the screening of their photos, describing why they took and selected specific photos, responding to questions from the researchers and also to questions and comments from the other participants. The discussion which followed provided a rich source of data, not only about individual participants' views about the role of everyday spaces in providing information and supporting learning, but also allowed participants to discuss similarities and differences. At the end of the focus groups, all groups came together and all the photos were displayed so that every participant could see the photos. The ensuing discussion between the participants and researchers was audio taped and formed part of the data bank.

The data from the focus group discussions and photographs were then drawn together to create a situational landscape map [28] using grounded theory approach [29] to present a composite picture of the common themes and perspectives about the types of information that were important, the ways in which the participants gained information and how this information supported their learning.

\section{Analysis of data}

Phase one of the analysis is reported in this paper. This analysis was undertaken using the visual images and transcribed interview data from the focus group sessions to create a map of the information landscape of refugee youth. A constant comparative technique [30] was employed where data is compared with data, and code and categories are compared to identify emergent categories [28]. This process enabled common themes and perspectives to emerge through this stage of the analysis. A situational map [28] was created which highlighted key sites, sources and tools that contribute to learning in everyday spaces.

A central finding of this analysis is that informal learning through everyday spaces is an important activity, which supports gaining entry to the broader Australian community and linking into networks. There were two activities revealed that were central to the young people becoming emplaced. These activities were: gaining entry to the 
community, to learn about how the information landscape is shaped; and learning how to link-in to access the embodied and embedded knowledges of community know-how, in addition to more normative sources that allowed young people to learn about how the community operates. Emplacement describes the practice of becoming situated, of being located and of locating, of knowing how the information landscape is shaped, learning how relevant sources of information are located, and how to enact information literacies and skills practices to develop a way of knowing how to go on.

\section{I. Everyday Spaces}

Everyday spaces are social and situated and are formed around particular types of activities (e.g., youth groups, playing sport, doing art); they therefore had an emotional resonance for participants. The spaces were categorised as group physical space (highlighting the physical co presence), socio-technical (thus highlighting the social use of technology) and institutional spaces. Connecting with these spaces enabled access to sources of formal and informal information. While formal sources will reference the discourse and narrative of the space, informal sources are tacit, secondary and nuanced, may not be easily expressed, remain tacit, or are only available contingently [31, 32]. Examples of each of the sites, commencing with 'group physical spaces' will be described below. Participants' names are pseudonyms.

\section{I.I. Church}

Attendance at church afforded participants the opportunity to meet and mingle with people from a wide range of walks of life. Church also built important social connections to connect with older members who could offer not only guidance on faith, but also other useful information based on expertise, such as that provided by:

...the guy that does the food at the end ... he helps me out cause he is one of the students at (the local university) and he talks to me about courses I can get into next year ... what I require to get into them and whether or not I can do it (locally) or not.

At church, the young people mixed with people from a myriad of social backgrounds:

...in the church you can find lawyers, you can find doctors; you can find all sorts of people. So giving you the opportunity to seek each and every information that you would like.

They learned about what jobs might be out there, and how to tap into the hidden job market, for example:

You can find employment through people there. Especially (in our local town) the best way to find a job is kind of who you know ... you can put it out there like I'm looking for a job and I have qualifications in what, what you know can you guys ask around for me and people are more than happy to do that.

\section{I.2. "Faith"-based groups}

Faith-based groups were represented in many of the photos taken by participants. This space acted as not only a place where young people could connect with their religious beliefs, but also as a space to connect with friends and seek advice and information at peer-peer level about issues that affected young people's lives, and that might not be spoken about with other family members. Friends were:

...important to me because they're my friends and I ask them any questions - like some questions you can't really ask your family or just random people - you just have to ask your friends and they help you like grow to a better person.

\section{I.3. Sporting teams}

Sport was a crucial part of the boys' lives. The three girls also loved sport but opportunities for them to be involved were more limited than for the boys. Some of the boys in the study were members of the town's organised teams. This provided them with key social connections amongst peers, older players and adult coaches whom they respected, trusted and went to for advice about personal issues:

(If you) really trust your coach and that and sometimes if you have trouble in love you know if you're close with, then they can help you out and give you certain information and certain stuff and making sure you know where to go get it from.

Through the sporting space, they connected with information, which allowed them to develop leadership, cooperation, and team-building skills that are important to resilience. The concept of equality and social inclusion was reinforced. The boys agreed that: 
...the other thing sports does too is break down racial barriers, that's very important when it comes to sports and you feel that everybody is your equal as they're judging ability, not necessarily your skin colour or where you come from.

Sometimes the boys got help with their homework from the older boys:

...if you come to footy and you still have to do assignments the boys will help you out and stuff too because some of the boys who play under 17 games are year SC doing their HSC trials and stuff.

Sport was a source of crucial information - they learned about jobs that were coming up or, if they wanted work, they let their teammates and coaches know and sometimes they got work that way.

\subsection{Socio-technical spaces}

Socio-technical sites reference both physical and virtual tools and media, linking the use of these sites to becoming informed about local and world events, finding out about work, and communicating with friends to share information. There was an emphasis on maintaining social connections and mediating on behalf of family. The Internet and social media represent a pervasive and seamless parallel space for refugee youth. This space is not viewed as separate or in addition to daily enterprise, but as integral and inseparable, providing a critical entry point that allowed interconnectivity and enabled participants to draw from local, national and global knowledges, for educational, social and familial purposes.

\subsection{Facebook}

Facebook was used for multiple reasons by participants and thus informed learning on many different levels. Participants recognised the dual purpose of Facebook as a source of push and pull information and of information sharing which contributed to their information landscapes. The complexity of the Facebook landscape was acknowledged by participants and many described knowing how to operationalise Facebook as an important literacy practice (digital literacy) which allowed them to connect with a wider range of social media resources and tools to connect with the virtual world. As one participant noted:

...Facebook itself, is not, it's not all about social media, it's also about knowing the site

The reason why I chose this [photo] is most people get information through Facebook and Facebook being the most general source of social media, just be rated good background... people, communication, relationships. Our friends and maybe getting, people, getting friends, finding about the world, is most of the things done on Facebook

When asked why a particular photo of Facebook was taken, a young female participant acknowledged the complexity of the source and its various links with other important social information sources:

It's important because I find a lot of stuff on Facebook the social media cause I'm on there every day and yeah there's like different stuff on there and so yeah.

\subsubsection{Gaming}

The information quality of gaming sites rests in the capacity to develop or improve skills and knowledge which support learning in real world situations. In describing a photo depicting game play, a participant described how the game helped him develop critical thinking and problem-solving skills that assisted his real-world learning. He reported that:

...it depends on the video games that have to do you have to find out clues and knowledge and stuff in video games and stuff like that and you have to go to different places find clues...

When asked if he meant problem solving, he stated:

Yeah, that's the word and helps you get information from different sources like we're doing right now...

Another participant described how his younger brother, now a State representative in basketball, connected with information about the rules and strategies of playing of basketball: 
After I started playing basketball he got into the basketball too and I bought the game and then he just played it 24/7 until one day

I just forgot and now he knows more than me

Learning was encountered as an activity that must be translated into real life situations- however the effectiveness of this information was measured in its ability to be replicated:

If he see it he tries it in the game and goes ah that's cool and I'm going to go and try that so he goes and tries it and yeah and actually does it on the field, but yeah it's pretty amazing

Another participant described how gaming allowed him to inform his learning

You learn about how the English revolution started...and all this kind of stuff and what happened back in history even though it gets sort of weird. It's not all fact some of it is fiction, but the game has storylines they show you exactly what happened...

When asked how he might check the accuracy and reliability of this information, the participant demonstrated that he recognized the need to be critical about information. He stated that:

Well the facts like it has a story line when it starts up and it has a whole summary thing that comes up at the start of the game and then when you play it has a part ...that pops up and those are actually the facts.

When asked if he might check the facts elsewhere or whether he would just use this information, he stated that he would check them, but then indicated that he would go to Wikipedia - an unreliable and unsubstantiated source. Gaming also promoted problem-solving and many of the young men in the study cited games such as Assassins' Creed and Call of Duty as affording them the opportunity to develop and refine their information seeking and problem solving skills.

The findings suggest that everyday spaces played a central role in the informal learning of young people who might be outside the formal schooling system. The findings also indicate why these spaces are important in relation to testing out of information, maintaining global networks and mediating virtual information in support of family and their information needs.

\subsubsection{Testing information}

For the young people in this study Facebook also acted as site to 'test out' opinions with peers or to engage with conversations about issues or facts which might inform learning. The ability to differentiate between the different types of information sources available on a single site required that participants develop information literacy competencies that would enable them to enact digital literacies in the online environment. This capacity was summed up as follows:

Well, sometimes when you look on Facebook it has like well, here's videos that are funny, but you can also look up videos about educational stuff or depends on what pages you like, there can be different videos you can watch and you can learn heaps of things from them, on some pictures comes up with facts and stuff.

\subsubsection{Maintaining global networks}

Refugee youth identified significant benefits of virtual space because of the capacity to maintain close familial networks with family who were not 'in country' or were dispersed across broad geographic distances. It helped them to stay connected with their home culture. All young people in the study cited Facebook as the primary mechanism for remaining connected to family who were either still in their original country or who had resettled in other locations:

Well my uncle he's in Africa and then when he calls on the phone the network cuts off and stuff so he emails me through Facebook and I can tell mum stuff so that's the easiest way yeah

\subsubsection{Mediating virtual spaces}

The complexity of the online information environment, and government policies of pushing information online, had placed young refugee youth in a position where they were often called upon to search for information on behalf of their immediate and extended families, who might not possess the information or digital literacies required to undertake these tasks confidently. This placed additional pressure on the participant group. It also highlights the importance of effective information literacy training to ensure that young people are equipped with sufficient information literacy competencies 
to ensure that the information they search for and then disseminate to others is accurate, credible and reliable and presents an even view rather than one influenced by age differences or perspectives.

All participants in the study identified that they were asked to find information on behalf of family - this was, however, more prevalent with female participants:

Mainly for myself and for my family members.

Oh sometimes if my mum needs something printed or she wants me to search something ...

The Internet was also searched to obtain information about global events that affected their homeland. Specific sites were bookmarked to ensure that a level of currency in relation to global issues could be maintained.

\subsection{Institutional Spaces}

All participants acknowledged the importance of institutional spaces as significant sources of formal information, but also as a source of local knowledge that enabled them to build their life skills:

You know that you can get help from any of the people that are here and if you just need a job they will help with that. Just get you sorted out ... even if you have like troubles or fines and stuff like that, random stuff you can come here talk to (the workers) or someone.

Sources of information (particularly people) were often used as a first port of call because personnel such as youth refugee service providers had spent a long time building trust and credibility with these young people.

In one youth network created by a major refugee service provider, a number of the young people learned leadership skills, how to work together as a team, the importance of taking it in turns to listen to each other's opinions and to discuss how to go about accomplishing necessary tasks:

We've learnt so much from it and from all the leadership lessons, relationships, because those kind of stuff you can't get that from somewhere else and you can't talk about relationship things with your parents if you know they're not going to, I don't know, you need other sources of information you know.

Mental health services for youth provided participants with information through counselling services and many were now strong advocates for this service, advising others about the types of information and support that could be provided. One participant in describing the mental health services recognised that:

...this is a good place for young people or people in general to get information and to get help.' I think especially the African community they need to get introduced more into this place

Health workshops assisted many of the young people to understand contemporary health issues and to discuss difficult topics such as alcohol, illicit drugs and relationship issues. These workshops provided useful information about the services that were available for friends who were suffering from depression or addicted to drugs or alcohol:

They do the health clinic ... one for depression and stuff like that. One that they did was what to do if say your friend - someone you are with - has like an overdose or something like that - the steps to do - that kind of stuff

\subsection{Libraries}

For young people in the study the library represented a source of knowledge and provided information about a wide range of topics. It was a safe and quiet space to study, to ask questions, and get advice. The librarians were an excellent source of information, knowledge and support for study. For one participant, books were not the only source of information, as the library also had guest speakers. . The speakers were described as: 
affect(ing) your way of understanding and to develop you.

Books also provided

...understanding, learning, and education

and when you entered the library:

...the information you're looking for is definitely there, meaning that it's also a good source of information

The library was viewed as providing a broad and balanced range of information sources. Described in the following way by a participant:

...there is many information that we cannot get it from internet or from mobile ... we can find movies on YouTube, we can get it in internet, but we cannot get books, many kind of books, interesting books, social ... that's why library important place to get books.

\section{Discussion: Information literacies and learning in everyday spaces.}

The use of the photovoice technique created the opportunity for participants to expressly illustrate how engaging with everyday spaces afforded opportunities to operationalize and support their learning. This in turn, allowed them to become emplaced in the community. Mapping the sites and sources of the information landscape references the social, virtual and institution spaces, and reflects ways of knowing that may be explicit, embodied or contingent depending on the space accessed. It also draws attention to the sites of silence [33, p.561] such as nuanced, local or contingent knowledges which are central to informal learning.

The photovoice technique also draws attention to information literacy not only as an educational practice but also as a social practice that is foundational to learning in everyday life. Information literacy is evidenced in this study, by the ability of participants to describe and demarcate the role of various spaces in contributing to learning and to operationalize this understanding through the enactment of appropriate contemporary literacies (digital, visual, media) relevant to the source and described as fit for purpose. Overall, the use of the photovoice technique enabled a more focused understanding of how and where information use and learning occur outside formal settings.

Everyday spaces were central to the building of an information landscape, affording opportunities to connect with information that may not be available in structured formal settings. To link-in and connect to everyday spaces, participants operationalized ways of knowing on two levels:

Level 1. Newcomers learned about the landscape's structure and about ways of knowing how the structure was shaped, e.g., what information resources were available to them as well as the types of knowledge and information that were sanctioned and legitimised. Newcomers learnt to navigate within the landscape (identifying which literacy practices were enacted), how information was operationalized, and how the space was entered. This is epistemological in nature; and,

Level 2. How people engaged with situated knowledge of the landscape (know-why knowledges). This is the ontological nature of the site.

Information landscapes were constructed and accessed by information literacy practices which were enacted within a setting and which reflected the sayings and doings of the discourse community [9]. In this present study identifying and then enacting literacy practices that supported gaining entry and linking-in to everyday spaces allowed participants to engage with learning how to:

- Map the landscape:

$\circ$ Make sense of the landscape, identifying where knowledge sites were located and the primary and secondary affordances that were offered;

- Engage in social participation:

- Establish and maintain social links

- Keep in touch

- Make contacts 
- Seek and access contingent information opportunities

- Make sense of new spaces; and

- Get organized and learn life skills:

- Find a job

$\circ \quad$ Connect and maintain interests (art, sport)

Emplacement was achieved by gaining entry to the community and linking in. It was supported by the practice of information literacy that acted as a foundational literacy supporting the construction of meaning by connecting refugee young people to information resources within everyday spaces, relevant ways of knowing and sources of information. To gain entry and link in requires knowledge about the information resources of an everyday space and how to operationalize access to the sources of and sites of knowledge that are important to the community. In the present study information literacy was therefore seen to be enacted through a wide range of contemporary literacies which allowed young people in the study to transition into their adopted communities. Implicit in the bridging of spaces were the affordances of opportunities to bond with the community. In other words, learning to access and draw from local knowledges such as their peer groups, situated the young people in this study within the same sematic and cultural space.

Everyday spaces such as faith-based groups, church and sporting teams, provided access to social, vernacular/local and tacit information sources that young people required to establish themselves within the wider community beyond the schoolgate. Online spaces such as Facebook were important in this capacity because they provided access to a wider range of information sources and sites of knowledge, thus extending the local social networks to include global networks [34] The use of these sites enabled participants to connect with information that confirmed their affinity with local networks, allowing them to learn and position themselves and establish new networks within their new community. Social media also supported and maintained global peer-peer relationships, particularly with those who had had similar experiences of relocation. Family connections were also maintained thus ensuring that the links with culture that may have become fragmented through movement were re-established [7, 35].

In general, online spaces have now become so entrenched and seamless, they are viewed by the young people in this study as simply 'another space' where everyday life existed and was ongoing. What became interesting was how the literacies that related to these spaces became enacted, as many of the participants had a clear view about the affordances offered by the spaces as being "fit for purpose". This suggests that participants evaluated the information qualities of particular sites and their information resources and made decisions about what literacies of information to enact, e.g., digital spaces require digital literacy to be enacted; social spaces such as church or sporting teams require other forms of literacy practice such as visual literacy or vernacular practice relative to the social setting, such as identifying who to ask, listening, or asking questions.

Finally, the construct of affordances that describes the opportunities offered by the landscape to engage with it [36] emerged as an important element in this study of information sources, literacies and learning. Affordances are often considered to be primary (specific to purpose); however in the present study, participants also recognized secondary affordances. This recognition was prefaced on the foundation of shared meaning [7]. Primary affordances related to the specific purpose of the site, e.g., churches were place to connect and renew faith. Secondary affordances related to opportunities that were contingent on the members of the site, e.g., church was referenced primarily as a place to connect with religious discourse and renewal of faith. However, the congregation after church provided other information affordances, i.e., getting advice about health, or finding out about potential employment.

Thus, participants were able to leverage the affordances of everyday spaces by accessing not only primary affordances but also accessing the hidden affordances within these sites that were often only available contingently. The idea of contingency of information provision was noted by Fisher, Durance and Hinton [11], leading these authors to locate contingency in the construct of information grounds. According to Fisher [36, p. 185] information grounds can be defined as "environment[s] temporarily created when people come together for a singular purpose, but from whose behaviour emerges a social atmosphere that fosters the spontaneous and serendipitous sharing of information."

Also highlighted was the vernacular or local literacy practice, which enables people to connect with social information that is tacit and often difficult to express in written form (e.g., knowing who to talk to about possible work, or information that might provide a clue about why things work in certain ways) [3]. Learning was described as a physical activity and supported by visual literacies, which require observation and repetition of particular tasks so they become an unconscious action and embedded as ways of knowing. 


\section{Conclusion: Implications for service providers}

Whilst further analysis is ongoing, a number of salient lessons can be learnt from this study of refugee youth and their engagement with the information landscape, which may be extended to other marginalised youth cohorts within the community. Refugee youth are faced with complex information needs (to become part of community, relate to peers, deal with social challenges that arise from cultural expectations, learn the rules of the social game; find employment; fulfil familial expectations, stay connected). These needs require them to identify and map the everyday spaces that can contribute not only to their learning but can also support the learning of their family whom they are required to support. One important challenge for service providers is to ensure that refugee youth are equipped with information-seeking skills so that the information they search for and then disseminate to others is accurate, credible and reliable and presents an even-handed view.

The challenges highlighted the importance of effective information literacy training for young people to ensure that young people outside school are equipped to make informed decisions by ensuring their learning is supported by information literacy and its enactments. Also highlighted was the need for service providers who support this cohort to be aware of how central learning in everyday spaces is to support refugee youth emplacement into the broader Australian community.

\section{Funding}

The Uniting Care Council Innovations Grant funded this project.

\section{References}

[1] Major J, Wilkinson J, Langat K, Santoro N. Sudanese young people of refugee background in rural and regional Australia: social capital and education success. Australian and International Journal of Rural Education. 2013; $23(3): 95$.

[2] Sampson R, Gifford SM. Place-making, settlement and well-being: the therapeutic landscapes of recently arrived youth with refugee backgrounds. Health \& Place. 2010;16(1):116-31

[3] Lloyd A. Information literacy landscapes: Information literacy in education, workplace and everyday contexts. Oxford, UK.: Chandos; 2010.

[4] Kemmis S, Wilkinson J, Edwards-Groves C, Hardy I, Grootenboer P, Bristol L. Changing practices, changing education: Springer Science \& Business Media; 2013.

[5] Lloyd A. Following the red thread of information in information literacy research: Recovering local knowledge through interview to the double. Library \& Information Science Research. 2014; 36 (2):99-105

[6] Fisher KE, Peterson Bishop A, Fawcett P, Magassa L. InfoMe; A field-design methodology for research on ethnic minority youth as information mediaries. New directions in Children's and Adolescents Information Behaviour Research: Emerald Publishing Group; 2014, (10):135-156.

[7] Lloyd A, Kennan MA, Thompson KM, Qayyum A. Connecting with new information landscapes: information literacy practices of refugees. Journal of Documentation. 2013; 69(1):121-44.

[8] Blumer H. Symbolic Interactionism: Perspective and method. Englewood Cliffs. N.J.: Prentice Hall; 1969.

[9] Bourdieu P. The forms of capital. In: Richardson JG, editor. Handbook of Theory and Research for the Sociology of Education New York,: Greenwood Press; 1986. pp. 241-58.

[10] Schatzki T. The site of the social: A philosophical account of the constitution of social life and change. Pennsylvania: Pennsylvania State University Press; 2002.

[11] Leckie G, Given L. Henri Lefebvre and Spatial Dialectics. In: Leckie G, Given L, Buschman J, editors. Critical theory for Library and Information Science. Santa Barbara, Calif: Libraries Unlimited; 2010. pp. 221-36.

[12] Fisher KE, Durrance JC, Bouch Hinton M. Information grounds and the use of need-based services by immigrants in Queens, New York: A context-based, outcome evaluation approach. Journal of the American Society for Information Science and Technology. 2004;55(8):754-66.

[13] Savolainen R. Everyday Information Practice: A social phenomenological perspective. Maryland, US: Scarecrow Press; 2008.

[14] Husserl E. General introduction to a pure phenomenology. (F. Kersten, Trans.). Boston: Kluwer Academic Publishers; 1982.

[15] Habermas J. The theory of communicative action: Lifeworld and system: A critique of functionalist reason. Boston: Beacon Press; 1987.

[16] Lefebvre H. The production of space. Trans D. Nicholson-Smith. Oxford: Blackwell; 1991.

[17] Schatzki TR. The timespace of human activity: On performance, society, and history as indeterminate teleological events: Lexington Books; 2010.

[18] Chattopadhyay S. Narrating everyday spaces of the resettled Adivasis in Sardar Sarovar. Population, Space and Place 2010;16:85-101. 
[19] Bateson G. Steps to ecology of mind. San Francisco: Jason Aronson Inc; 1972.

[20] Lloyd A. Information literacy landscapes: an emerging picture. Journal of Documentation. 2006; 62 (5):570-83.

[21] Gergen K. Social construction in context. London: SAGE Publications; 2001.

[22] Ingold T. The temporality of the landscape. World Archaeology. 1993; 25(2): 152-74.

[23] Nardi B, O'Day L. Information ecologies; Using technology with heart. 1999. Boston: MIT Press.

[24] Julien H, Given LM, Opryshko A. Photovoice: A promising method for studies of individuals' information practices. Library \& Information Science Research. 2013; 35(4): 257-263.

[25] Wang C, Burris MA. Photovoice: Concept, Methodology, and Use for Participatory Needs Assessment. Health Education \& Behavior. 1997; 24(3): 369-87.

[26] Wang C. Youth Participation in Photovoice as a Strategy for Community Change. Journal of Community Practice. 2006;14(12):147-61.

[27] Pickard A. Research methods in information. London: Facet Publishing; 2012.

[28] Clarke AE. Situational Analysis: Grounded theory after the postmodern turn. Thousand Oaks, California: Sage Publications; 2005.

[29] Charmaz K. Constructing Grounded Theory; A practical guide through qualitative analysis. London: Sage Publications; 2006.

[30] Charmaz K. Constructing Grounded Theory. 2nd ed. London: Sage Publication; 2014

[31] Barton D, Hamilton M. Local Literacies: Reading and writing in one community. Oxon: Routledge; 1998.

[32] Blackler F. Knowledge, Knowledge work and organizations: An overview and interpretation. Organization studies. $1995 ; 16$ (6):1021-46.

[33] Clarke AE. Situational Analyses: Grounded Theory Mapping After the Postmodern Turn. Symbolic Interaction. 2003; 26(4):553-76.

[34] Gilhooly D, Lee E. The Role of Digital Literacy Practices on Refugee Resettlement. Journal of Adolescent \& Adult Literacy. 2014; 57(5):387-96

[35] Wilding R. Mediating culture in transnational spaces: An example of young people from refugee backgrounds. Continuum. 2012;26(3):501-11.

[36] Gibson JJ. The theory of affordances. In: Shaw R, Bransford J, editors. Perceiving, Acting and Knowing. Hilldale: Lawrence Erlbaum; 1977. pp. 67-82.

[37] Fisher KE. Information Grounds. In: Fisher KE, Erdelez S, McKechnie L, editors. Theories of Information Behaviour. Medford, NJ: Information Today; 2006. 